\title{
Breakfast high in whey protein or carbohydrates improves coping with workload in healthy subjects
}

\author{
Nora Sihvola $^{1}$, Riitta Korpela ${ }^{1}$, Andreas Henelius ${ }^{2}$, Anu Holm²,3, Minna Huotilainen ${ }^{2}$, Kiti Müller ${ }^{2}$, \\ Tuija Poussa $^{4}$, Kati Pettersson ${ }^{2}$, Anu Turpeinen ${ }^{5}$ and Katri Peuhkuri ${ }^{1 *}$ \\ ${ }^{1}$ Institute of Biomedicine, Medical Nutrition Physiology, Pharmacology, University of Helsinki, PO Box 63, \\ Helsinki FIN-OOO14, Finland \\ ${ }^{2}$ Finnish Institute of Occupational Health, Brain and Technology Team, Topeliuksenkatu 41 aA, \\ Helsinki FIN-00250, Finland \\ ${ }^{3}$ Department of Clinical Neurophysiology, Hospital District of Satakunta, Sairaalantie 3, Pori FIN-28500, Finland \\ ${ }^{4}$ Stat-Consulting, Vabverokatu 6, Nokia FIN-37130, Finland \\ ${ }^{5}$ Valio Limited, PO Box 30, VALIO, Helsinki FIN-O0039, Finland \\ (Submitted 6 September 2012 - Final revision received 12 February 2013 - Accepted 12 February 2013 - First published online 16 April 2013)
}

\begin{abstract}
Dietary components may affect brain function and influence behaviour by inducing the synthesis of neurotransmitters. The aim of the present study was to examine the influence of consumption of a whey protein-containing breakfast drink $v$. a carbohydrate drink $v$. control on subjective and physiological responses to mental workload in simulated work. In a randomised cross-over design, ten healthy subjects (seven women, median age 26 years, median BMI $23 \mathrm{~kg} / \mathrm{m}^{2}$ ) participated in a single-blinded, placebo-controlled study. The subjects performed demanding work-like tasks after having a breakfast drink high in protein (HP) or high in carbohydrate (HC) or a control drink on separate sessions. Subjective states were assessed using the NASA Task Load Index (NASA-TLX), the Karolinska sleepiness scale (KSS) and the modified Profile of Mood States. Heart rate was recorded during task performance. The ratio of plasma tryptophan (Trp) to the sum of the other large neutral amino acids (LNAA) and salivary cortisol were also analysed. The plasma Trp:LNAA ratio was $30 \%$ higher after the test drinks HP (median $0 \cdot 13(\mu \mathrm{mol} / \mathrm{l}) /(\mu \mathrm{mol} / \mathrm{l})$ ) and $\mathrm{HC}($ median $0 \cdot 13(\mu \mathrm{mol} / \mathrm{l}) /(\mu \mathrm{mol} / \mathrm{l})$ ) than after the control drink (median $0 \cdot 10(\mu \mathrm{mol} / \mathrm{l}) /(\mu \mathrm{mol} / \mathrm{l})$ ). The increase in heart rate was smaller after the HP (median 2.7 beats/min) and HC (median 1.9 beats/min) drinks when compared with the control drink (median 7.2 beats/min) during task performance. Subjective sleepiness was reduced more after the HC drink (median KSS - 1.5) than after the control drink (median KSS - 0.5). There were no significant differences between the breakfast types in the NASA-TLX index, cortisol levels or task performance. We conclude that a breakfast drink high in whey protein or carbohydrates may improve coping with mental tasks in healthy subjects.
\end{abstract}

Key words: Mental workload: Whey protein: Low energy: Breakfast

The modern work environment requires working on multiple simultaneous tasks under time pressure. This causes mental workload defined as the portion of an individual's limited mental capacity that is actually required by task demands, $^{\text {(1) }}$. In addition to task demands, factors such as poor diet and lack of sleep can decrease the physiological resources an individual has available for carrying out a certain task, and this can thus also increase the mental workload of the task. High workload during task performance may increase irritability, fatigue and facilitate errors ${ }^{(2)}$.
In extreme cases, high workload may contribute to stress and symptoms of work fatigue, sleep problems and depression ${ }^{(2)}$.

Brain function is thought to be very sensitive to even small changes in plasma metabolites ${ }^{(3)}$, and thus the brain energy supply and dietary components providing essential precursors to the brain may have an important role in optimising work performance and also in promoting mental well-being. Dietary interventions so far support the importance of having a breakfast, rather than skipping it, as this habit has been linked with several positive health outcomes, and also with improved

Abbreviations: 5-HT, 5-hydroxytryptamine (serotonin); B@W, Brain@Work; HC, high carbohydrate; HP, high protein; IQR, interquartile range; KSS, Karolinska sleepiness scale; LNAA, large neutral amino acid; NASA-TLX, NASA Task Load Index; POMS, Profile of Mood States; SS, Symmetry Span; Trp, tryptophan; WS, work simulation. 
cognitive functions, particularly in the elderly ${ }^{(4)}$ and in schoolaged children ${ }^{(5)}$. The type of breakfast also makes a difference since macronutrient composition, energy supply and glycaemic properties of the breakfast may all affect cognitive performance $^{(6)}$. In healthy work-aged adults, however, clear evidence on the necessity of breakfast is still lacking ${ }^{(7)}$ as is the consensus on which kind of breakfast would benefit the most in the work environment.

The availability of an essential amino acid, tryptophan (Trp) to brain serotonin (5-hydroxytryptamine; 5-HT) synthesis, is important for the regulation of, for example, stress adaptation, cognition and $\operatorname{mood}^{(8)}$. There are two nutritional ways to increase the level of Trp in the brain: through consumption of carbohydrates or Trp-rich whey protein ${ }^{(9)}$. Carbohydrates induce the synthesis of 5-HT through insulin response. Insulin secretion facilitates the precursor of 5-HT, Trp, to cross the blood-brain barrier for 5-HT synthesis, while the other large neutral amino acids (LNAA) are transferred into the skeletal muscle ${ }^{(10)}$. In addition to 5 -HT regulation, glucose is a primary source of energy for the brain and its beneficial effects on short-term performance have been recognised widely ${ }^{(11,12)}$. In a study by Markus ${ }^{(13)}$, a pure carbohydrate-containing breakfast drink improved cognitive performance and mood under stress. Carbohydrate-rich, protein-poor foods may also reduce levels of the stress hormone cortisol following acute stress-inducing tasks, but this has been shown only in stressvulnerable subjects $^{(14)}$.

Milk-derived whey protein, and particularly its $\alpha$-lactalbumin protein fraction, also increases the level of Trp in the brain by increasing the ratio of plasma Trp to the sum of LNAA (Trp:LNAA). A breakfast enriched with whey protein was proven to positively affect mood and performance in specific cognitive tasks in stress-vulnerable subjects in an acute stress test ${ }^{(15-18)}$. The effects of $\alpha$-lactalbumin on cognition are generally modest in healthy subjects ${ }^{(9)}$.

When comparing high-carbohydrate $v$. high-protein breakfasts in healthy participants, Lemmens et al. ${ }^{(19,20)}$ found no difference between the breakfast types in mood or cortisol responses in an acute stress and rest condition. Fischer et $a{ }^{(3)}{ }^{(3)}$ suggested that a protein-rich or balanced meal with a lower glycaemic index results in better overall cognitive performance than a meal rich in a mix of carbohydrates presumably because of less variation in plasma glucose and insulin concentrations and the glucagon:insulin ratio. Regarding cognitive performance, it seems that the changes in plasma metabolites are more important than the absolute levels ${ }^{(21)}$. Smaller blood glucose fluctuations result in lighter metabolic stress, which may be better for cognition ${ }^{(21,22)}$.

In previous studies, little consideration has been given to mental workload such as frustration and mental effort. However, these dimensions may be key parameters when evaluating the effects of breakfast or its macronutrient content on work performance. Under high workload that challenges cognitive resources, performance effectiveness may decrease, and, in addition, psychophysiological activation, strain and fatigue increase, even if the primary work task performance shows no decrement ${ }^{(23)}$. Thus, the aim of the present study was to compare the influence of consumption of a whey protein-containing breakfast drink $v$. a carbohydrate breakfast drink $v$. control on subjective and physiological responses to mental workload in simulated work. We measured mental workload and the changes in subjective well-being and sleepiness as well as heart rate in a computerised work simulation (WS). The cognitive demands of the tasks resemble the challenges that a real multitasking work environment places on attention and working memory ${ }^{(24,25)}$.

\section{Methods}

\section{Subjects and study design}

We studied healthy Finnish men and women ( $n$ 10, seven women) with a median age of 26 (range 22-40) years and a BMI of 23 (range $20-28$ ) kg/m $\mathrm{m}^{2}$ in a cross-over study. Exclusion criteria for participation were the use of medication (except use of contraception), diseases of the central or autonomic nervous system and also metabolic, hormonal or intestinal diseases, depression, overweight (BMI $>30 \mathrm{~kg} / \mathrm{m}^{2}$ ) or underweight (BMI $<18 \mathrm{~kg} / \mathrm{m}^{2}$ ), excessive use of alcohol (>240g alcohol in a week), smoking, food allergies (e.g. milk), lactose or gluten intolerance, pregnancy or breastfeeding. All subjects were habitual breakfast eaters, their sleep normally occurring between 21.00 and 08.00 hours and they did not consume caffeine more than $1000 \mathrm{mg} / \mathrm{d}$. Volunteers for the study were recruited via email and notice-board announcements from the campus of the University of Helsinki and from local companies. The present study was conducted according to the guidelines laid down in the Declaration of Helsinki, and the test protocol was approved by the ethics committee of the Hospital District of Helsinki and Uusimaa. Written informed consent was obtained from all subjects.

The study was conducted according to a randomised, single-blinded, cross-over design. Each subject visited the Brain Work Laboratory at the Finnish Institute of Occupational Health four times. The outline of the study is presented in Fig. 1(a). During the first visit, subjects became familiar with the experimental procedure. They were introduced to the laboratory surroundings and the individual difficulty level of the WS tasks was set as described in detail later in this section. Subjects were also introduced to the use of food diary, sleep diary and actigraphy, and they were trained to fill in the questionnaires on the computer. Subjects did not, however, consume the breakfast drinks during this visit. All four visits were separated by at least 1 week.

On the three actual test visits, subjects came to the laboratory at 08.30 hours, after fasting overnight, and received as a breakfast once a high-protein (HP), once a high-carbohydrate (HC) and once a control drink (control) and performed work-like tasks in the WS. The test drinks HP and HC were indistinguishable from each other, whereas the control drink was of different appearance. The investigators were blind to the HP and HC breakfast drinks. Although all of the breakfast drinks were not of identical appearance, the subjects were unaware of the content of the breakfast drinks. The order of the drinks was randomised across the subjects. The subjects had free access to water during the entire study, but no 
(a)
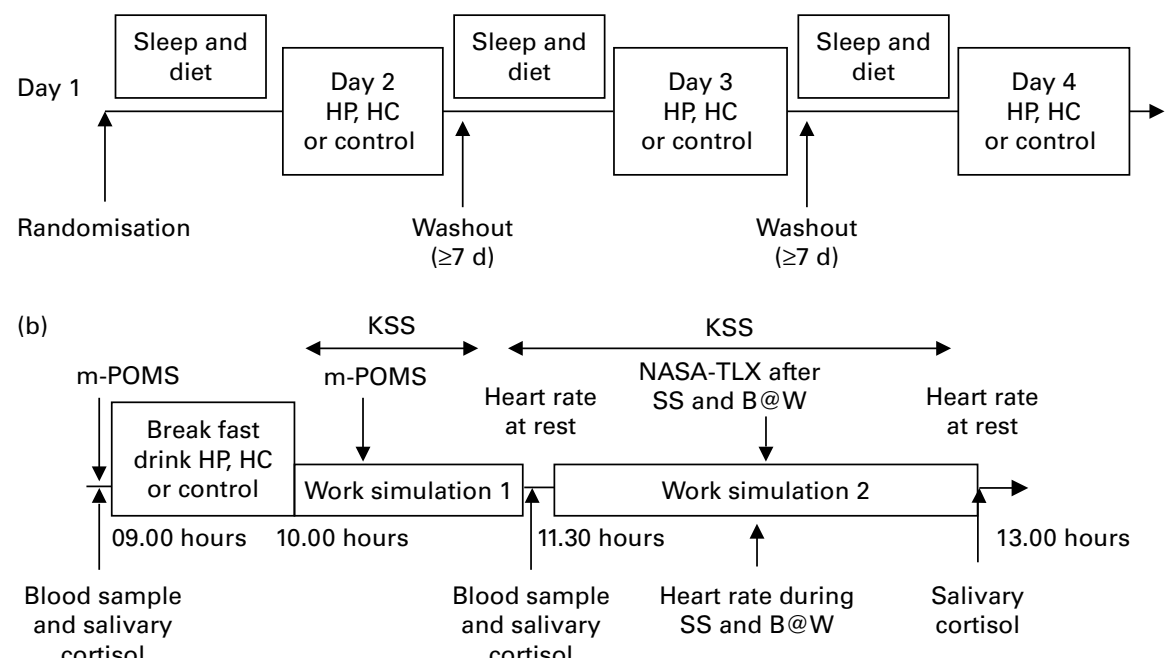

Fig. 1. (a) Study design and (b) schedule during the study days $2-4$ of the randomised $3 \times 3$ cross-over study to compare breakfasts with high protein (HP), high carbohydrate $(\mathrm{HC})$ and control which were given in a randomised order. Sleep and dietary information was recorded before each test session. Work simulations 1 and 2 lasted for 60 and 90 min, respectively. During work simulation 2, the Symmetry Span (SS) test and Brain@Work (B@W) multitask test were performed, respectively. KSS, Karolinska sleepiness scale; m-POMS, modified Profile of Mood States; NASA-TLX, NASA Task Load Index.

additional food or fluid intake was permitted. The schedule during test days $2-4$ is presented in Fig. 1(b).

After arrival in the laboratory on an actual test visit, the subject rinsed his or her mouth with water $15 \mathrm{~min}$ before the taking of a salivary sample. Information regarding the current health of the subject was asked. The subject filled in a baseline mood questionnaire, modified Profile of Mood States (POMS), which has been described in detail later on, and a baseline blood sample was taken after which the subject received one of the breakfast drinks at 09.00 hours. The dietary intake was supervised to ensure that the test drinks were consumed within $10 \mathrm{~min}$. After the breakfast drink consumption, electrodes for electroencephalographic recordings were placed on the scalp. The results of these recordings will be reported separately.

At 10.00 hours, the subject was seated in a silent measurement room to start WS1. This simulation, lasting for $60 \mathrm{~min}$, worked as a run-in session for the afternoon WS2 to standardise the physiological and psychological states of the subject. During WS1, the subject first performed a battery of simple attention and working memory tasks and also a more challenging computerised multitask test, Brain@Work (B@W). Subjective sleepiness was assessed every $10 \mathrm{~min}$ by the Karolinska sleepiness scale (KSS) on a computer. The subject's mood was assessed by the modified POMS for the second time during WS1 (at least 60 min after the breakfast drink consumption).

Earlier studies have suggested that the effects of macronutrients occur clearest in challenging cognitive circumstances and after a delay rather than immediately after the breakfast ${ }^{(12)}$. In addition, as we assumed that the effects of nutritive breakfast drinks would mediate through the changes in plasma Trp:LNAA and as it has been suggested that peak changes in Trp:LNAA are found $120-200 \mathrm{~min}$ after carbohydrate intake when glucose levels have returned to baseline ${ }^{(13)}$, blood samples were collected $120 \mathrm{~min}$ after the breakfast. Also, the second salivary sample was collected at this point.

After blood and salivary sampling and a short break, WS2 started at 11.30 hours and lasted altogether $90 \mathrm{~min}$. First, subjects were seated in a silent measurement room to first watch a silent nature documentary without subtitles for $5 \mathrm{~min}$. The purpose of the documentary was to standardise a baseline condition for the heart rate recordings. During the second battery of cognitive tests, subjects performed the same attention and working memory tasks and in the same order as during WS1. They additionally completed a complex span task, the Symmetry Span (SS), which was performed before the B@W.

In WS2, heart rate was recorded during B@W performance and subjects assessed their sleepiness every $10 \mathrm{~min}$ and also their mental workload after the SS and B@W tasks. At the end of the WS2, the subjects watched the second nature documentary during which the heart rate was recorded to find out the recovery of the heart rate. The third salivary sample was taken after WS2. The test day finished at about 13.00 hours when the subjects were provided a lunch. All the three test visits followed the same protocol as described here.

Subjects were asked to avoid alcohol and strenuous physical exercise the day before each testing day. Nutritional habits and sleep quality were recorded before each study session (Fig. 1(a)). Each subject was given a sleep diary, a wrist actigraphy (Actiwatch AW7; CamNtech) and a food diary in which they reported all the foods and drinks that they consumed (analysed with Nutris Plus; Healthmakers). The subjects were instructed to record information about their food intake for $1 \mathrm{~d}$ and their sleep for $2 \mathrm{~d}$ before the laboratory visits. Sleep time, sleep efficiency (the part of actual sleep time of the time spent in bed) and morning 
Table 1. Sleep and dietary information before the test sessions ${ }^{\star}$

(Medians, minimum and maximum values)

\begin{tabular}{|c|c|c|c|c|c|c|}
\hline & \multicolumn{2}{|c|}{ High protein } & \multicolumn{2}{|c|}{ High carbohydrate } & \multicolumn{2}{|r|}{ Control } \\
\hline & Median & Minimum-maximum & Median & Minimum-maximum & Median & Minimum-maximum \\
\hline Sleep time (h:min) & 7:07 & $05: 37-07: 58$ & $7: 07$ & $04: 46-08: 07$ & $7: 12$ & $05: 45-07: 56$ \\
\hline Sleep efficiency† (\%) & $90 \cdot 3$ & $80 \cdot 9-93 \cdot 6$ & 91.9 & $84 \cdot 8-95 \cdot 2$ & 89 & $72 \cdot 75-94 \cdot 1$ \\
\hline Sleepiness & $2 \cdot 8$ & $1.5-4.0$ & $2 \cdot 8$ & $2 \cdot 0-4 \cdot 5$ & 3 & $1 \cdot 0-5 \cdot 0$ \\
\hline Energy (kJ) & 7209 & $5481-11506$ & 7590 & $5042-12263$ & 8364 & $5941-12121$ \\
\hline Protein (E\%) & 19 & $9-24$ & 18 & $9-23$ & 22 & $11-30$ \\
\hline Carbohydrate (E\%) & 51 & $36-56$ & 45 & $23-53$ & 48 & $34-69$ \\
\hline \multicolumn{7}{|l|}{ Coffee $(\mathrm{mg} / \mathrm{d})$} \\
\hline Users $(n)$ & & 10 & & 9 & & 9 \\
\hline Minimum-maximum & & $50-600$ & & $100-700$ & & $100-600$ \\
\hline \multicolumn{7}{|l|}{ Alcohol (g/d) } \\
\hline Users $(n)$ & & 1 & & 3 & & 2 \\
\hline Minimum-maximum & & 12 & & $12-84$ & & $15-24$ \\
\hline
\end{tabular}

$\mathrm{E} \%$, percentage of total energy

* Sleep variables (actigraphy and sleep diary) are based on two previous nights and dietary variables are based on the previous day before the test sessions with the breakfast drinks of high protein, high carbohydrate and control in a $3 \times 3$ cross-over trial $(n 10)$.

$\dagger$ The part of actual sleep time of the time spent in bed.

$\ddagger$ Subjective assessment after an overnight sleep on a scale from 1 (very well rested) to 5 (not rested at all).

sleepiness (scale from $1=$ very well rested to $5=$ not rested at all) and dietary parameters (energy, protein and carbohydrate intake, coffee and alcohol use) assessed before the test sessions are shown in Table 1 . These variables did not differ significantly between the three test sessions.

\section{Breakfast drinks}

On the three experimental mornings, the subjects received fruit juice drinks as their breakfast. The two nutritive breakfast drinks were similar in energy content, appearance and taste with the exception of the nutrient composition: the proteinenriched drink contained $21 \mathrm{~g}$ whey protein (Davisco Foods International) and the carbohydrate-enriched drink contained $21 \mathrm{~g}$ maltodextrin (Cargill). The nutritive drinks were prepared by mixing the whey protein or the maltodextrin powder and $500 \mathrm{ml}$ of fruit juice (a mix of orange, grape, peach, mango and passion; Valio Limited). The energy content of the carbohydrate and protein drinks $(1255 \mathrm{~kJ})$ was at the same level with the habitual energy intake of Finns for a morning ${ }^{(26)}$.

The control drink contained artificially sweetened fruit juice without almost any energy $(67 \mathrm{~kJ} / 500 \mathrm{ml})$. It was prepared by mixing $50 \mathrm{~g}$ of artificially sweetened fruit juice concentrate (Felix Abba Limited) and $450 \mathrm{~g}$ of water. The energy, protein and carbohydrate contents of the drinks are given in Table 2.

\section{Mental workload}

The NASA Task Load Index (NASA-TLX) is a measure of subjective perception of workload ${ }^{(27)}$. Subjective mental workload was measured on a computer after the performance of cognitive work-like tasks (SS and $\mathrm{B} @ \mathrm{~W}$ ) during WS2. The NASA-TLX assesses six dimensions of workload (mental, physical and temporal demand, performance, effort and frustration) on visual analogue scales ranging from not at all (0) to very much (100). Mental workload was calculated as a mean score of six dimensions.

\section{Sleepiness}

Subjective sleepiness was measured on a computer by the nine-point $\mathrm{KSS}^{(28)}$. The scale varies from very alert (score 1) to very sleepy, fighting sleep or high effort to keep awake (score 9). Sleepiness was calculated as a mean score from the measurements registered every $10 \mathrm{~min}$ before and during WS2. The change in sleepiness was calculated by subtracting the mean KSS scores before WS2 from the mean scores during WS2.

\section{Mood states}

Changes in mood were measured on a computer using a validated Finnish version of the POMS ${ }^{(29)}$, which is comprised of seven different subscales for negative mood: tension, fatigue, obliviousness, depression, irritability, inefficiency, insecurity and vigour as a subscale for positive mood. All the subscales consisted of three to six negative or positive feelings. We modified the POMS by adding a subscale for the feeling of well-being. It referred to postprandial wellness and comprised the feelings of pleased, relaxed, vigour and satisfied. The subscale of well-being is based on the questionnaire used in the study of Boelsma et $a l .^{(30)}$. In addition, the original self-rating scales were changed to visual analogue scales ranging from not at all (0) to very much (100).

Table 2. Nutritional contents of the breakfast drink portions $(500 \mathrm{ml})$

\begin{tabular}{lccc}
\hline & High protein & High carbohydrate & Control \\
\hline Energy $(\mathrm{kJ})$ & 1255 & 1255 & 67 \\
Carbohydrates $(\mathrm{g})$ & 55 & 76 & 4 \\
Sugars & 55 & 55 & 4 \\
Maltodextrin & 0 & 21 & 0 \\
Protein $(\mathrm{g})$ & 21 & 0 & 0 \\
Whey protein & 21 & 0 & 0 \\
\hline
\end{tabular}




\section{Computerised work-like tasks}

High mental workload was induced with two computerised tasks: B@W, which demands working memory and divided attention $^{(31)}$, and SS, which demands visuospatial working memory ${ }^{(32)}$. The $\mathrm{B} @ \mathrm{~W}$ multitask has been developed at the Finnish Institute of Occupational Health and is a modification of SYNWORK, computer-based software for the assessment of performance in a simulated work environment ${ }^{(33)}$. The $\mathrm{B} @ \mathrm{~W}$ allows the task difficulty and pace to be adjusted ${ }^{(25)}$. In the $\mathrm{B} @ \mathrm{~W}$ task, four different simultaneous subtasks were used: an auditory attention task; an arithmetic task; a memory task; a visual vigilance task. On the first visit in the laboratory, subjects performed the B@W until a constant performance level was reached with an individual task difficulty level. The level of the subtask difficulty and the timing of the subtasks (stimulus display time and inter-stimulus interval) were defined so that the subjects were able to obtain approximately $70 \%$ of the maximum positive score. The individual task difficulty is very important since it is a means to avoid interferences of learning with treatment effects and to ensure that the task demands are similar for each subject and for each of the three test days.

\section{Heart rate}

Cognitive workload $^{(34)}$ and mental stress $^{(35)}$ activate the sympathetic nervous system and can thus be evaluated by measuring the heart rate. Heart rate was evaluated from the electrocardiogram during a $20 \mathrm{~min}$ task simulating work $(\mathrm{B} @ \mathrm{~W})$, and at rest during the viewing of a 5 min nature documentary shown before and after WS2. The electrocardiogram was recorded using a NeurOne amplifier (Mega Electronics Limited) and heart rate was calculated using the Matlabbased Biosignals analysis package ${ }^{(36)}$. The change in heart rate was evaluated in two ways: by subtracting the mean heart rate at rest before WS2 from the mean heart rate during the $\mathrm{B} @ \mathrm{~W}$ and by subtracting the mean heart rate at rest after WS2 from the mean heart rate during the B@W.

\section{Biochemical measurements}

Blood samples were taken twice during each test session: in the morning after the overnight fast and $2 \mathrm{~h}$ after the breakfast (before WS2). Plasma for analyses of the total Trp concentration and its ratio to other LNAA (valine, isoleucine, leucine, tyrosine and phenylalanine) was collected in $5 \mathrm{ml}$ evacuated tubes containing sodium heparin (Venosafe $^{\mathrm{TM}}$ heparin tubes; Terumo Europe N.V.).

Salivary cortisol samples were collected three times during the test sessions: in the morning and before and after WS2 with the Salivette sampling device (Sarstedt). Subjects were instructed to gently chew the cotton swab for $1 \mathrm{~min}$. The cotton swabs were stored in Salivette tubes in the fridge before being centrifuged at the end of the day at $1000 \mathrm{rpm}$ for $3 \mathrm{~min}$. The Salivette tubes were stored at $-20^{\circ} \mathrm{C}$ until the salivary cortisol concentrations were measured with liquid chromatography-tandem MS.

\section{Data analysis}

Primary variables measuring psychological coping with mental workload were the indices calculated from the NASA-TLX, KSS and modified POMS questionnaires. Physiological variables (heart rate, salivary cortisol levels and plasma Trp:LNAA) as well as work performance were secondary variables reflecting mental workload. As the effects of the diet have not been investigated in the Finnish Institute of Occupational Health's WS, the sample size was estimated based on other studies using the same model ${ }^{(25,31,37)}$. Data are expressed as medians with interquartile ranges (IQR) and minimum and maximum values. As the assumptions for parametric tests were not met, non-parametric Friedman's ANOVA was used as a global test to compare the breakfast drinks HP, HC and control. In the case of a significant global $P$ value, post hoc pairwise comparisons were performed using the Wilcoxon signed-rank test. All three pairwise comparisons were conducted: HP $v$. control; HC $v$. control; HP $v$. HC. Within-subject changes (during breakfast $v$. before breakfast) were analysed, when appropriate, such as for mood and biochemical variables. Within-subject changes during work-like tasks were analysed for the KSS score and heart rate. $P$ values of less than 0.05 were considered as statistically significant. Statistical analyses were performed with PASW Statistics 18 software (SPSS, Inc.).

Table 3. NASA Task Load Index (visual analogue scale $0-100 \mathrm{~mm}$ ) measured after the two successive work-like computerised tasks (Symmetry Span (SS) test and Brain@Work (B@W) multitask test) with the breakfast drinks of high protein, high carbohydrate and control in a $3 \times 3$ cross-over trial $(n 10)$

(Medians, interquartile ranges (IQR), minimum and maximum values)

\begin{tabular}{lcccc}
\hline & High protein & High carbohydrate & Control & $P$ (Friedman's ANOVA) \\
\hline SS & & & & \\
$n$ & 10 & 9 & 10 & 0.46 \\
Median & 59 & 57 & 61 & \\
IQR & $46-67$ & $39-60$ & $49-66$ & 0.67 \\
Minimum-maximum & $15-69$ & $0-68$ & $17-66$ & 10 \\
B @ & 10 & 10 & 53 & \\
$n$ & 47 & 48 & $39-67$ & \\
Median & $33-66$ & $34-62$ & $24-74$ & \\
IQR & $24-76$ & $6-77$ & & \\
Minimum-maximum & & &
\end{tabular}




\section{Results}

\section{Mental workload}

As shown in Table 3, during work-like tasks, subjective mental workload did not differ significantly between the breakfast conditions.

\section{Sleepiness}

Before WS2, the subjective feeling of sleepiness was at the same level after all breakfast drinks: the median KSS value was 6.0 (minimum-maximum 4-8) after the HP drink, 6.0 (minimum-maximum 4-8) after the HC drink and 6.0 (minimum-maximum 4-9) after the control drink. During WS2, a significantly different (Friedman's ANOVA $P=0.045$ ) median change in sleepiness was observed between the breakfasts with the HC $(-1.5$ (IQR -2.0 to -0.13$)$, HP ( -0.4 , (IQR $-1.3-0.28)$ and control (-0.5 (IQR $-1.5-0.79)$ drinks. Pairwise comparisons showed that there was a significant difference between the HC and control drinks $(P=0 \cdot 021)$, but no differences between the HP and control drinks $(P=1.00)$ and the HC and HP drinks ( $P=0.086)$. The results are shown in Fig. 2.

\section{Mood states}

The feeling of well-being reduced significantly after the control drink compared with the HP drink (Wilcoxon signed-rank test $P=0 \cdot 028)$, but there was no significant difference between the $\mathrm{HC}$ and control drinks $(P=0.36)$ or the HC and HP drinks $(P=0.95)$. The median change was -4.4 (IQR-10.0-4.4) after the HP drink, -5.0 (IQR - 15.6-5) after the HC drink and -13.1 (IQR -23.8 to -3.4 ) after the control drink on a scale of $0-100$. There were no significant differences between the breakfast drinks in the changes of mood subscales: tension, fatigue, obliviousness, depression, irritability, inefficiency, insecurity and vigour.

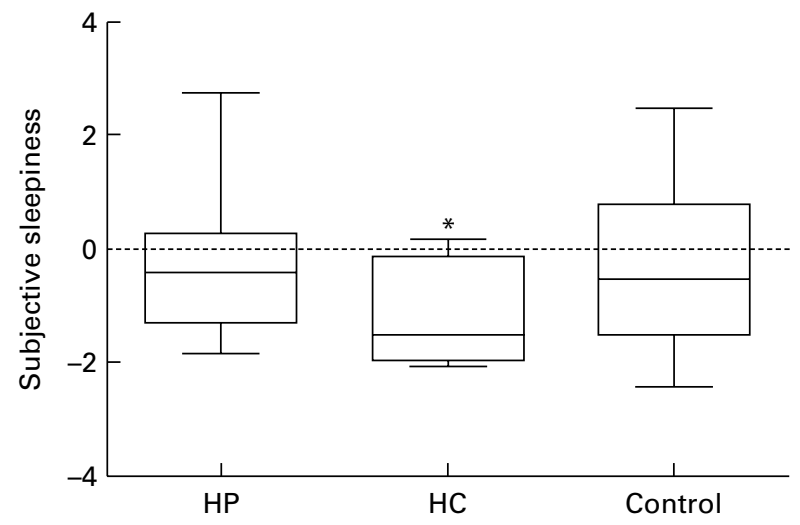

Fig. 2. Change in subjective sleepiness $(1=$ very alert to $9=$ very sleepy $)$ when sleepiness during work simulation 2 was compared with sleepiness before work simulation 2 after a high-protein (HP) drink, high-carbohydrate (HC) drink and control breakfast. Boxes indicate the lower and upper quartiles and the central line is the median. The upper and lower bars indicate the maximum and minimum values, respectively. There was a significant difference between the study breakfasts (Friedman's ANOVA $P=0.045$ ). * The HC drink decreased sleepiness significantly compared with the control breakfast $(P=0.021, n 10)$.

\section{Computerised work-like tasks}

Performance in the B@W or SS measured as total points, maximum points and correct answers did not differ significantly between the breakfast conditions (data not shown).

\section{Heart rate}

Heart rate increased during the $\mathrm{B} @ \mathrm{~W}$ multitask test after the HP drink (from median 63 (IQR 61-69) to 69 (IQR 63-72) beats/min), after the HC drink (from median 61 (IQR 59-68) to 66 (IQR 62-70) beats/min) and after the control drink (from median 59 (IQR 58-63) to 65 (IQR 64-72) beats/min). The median increase was $2 \cdot 7$ (IQR 0.03-6.4), 1.9 (IQR - 1.5-5.3) and $7 \cdot 2$ (IQR 4.5-8.7) beats/min for the HP, HC and control drinks, respectively (Friedman's ANOVA $P=0.030$ ). Pairwise comparisons yielded a significant difference between the HP $v$. control drink $(P=0.017)$ and between the HC $v$. control drink $(P=0.025)$, but not between the HP and HC drinks $(P=0.67)$. Thus, the heart rate increased more after the control breakfast when compared with the HP and HC drinks. The results are shown in Table 4 . Heart rate after the breakfast drinks did not differ significantly when comparing changes during and after the multitask.

\section{Biochemical measurements}

As shown in Table 5, $2 \mathrm{~h}$ following the breakfast, the plasma Trp:LNAA ratio and the change in the Trp:LNAA ratio between before and after the breakfast were significantly higher after the HP and HC drinks $(P<0 \cdot 001)$ when compared with the control drink. The HP and HC drinks increased plasma Trp:LNAA by $30 \%$ from the baseline when compared with the control drink. There was no difference between the study sessions in Trp concentration and Trp:LNAA after the overnight fast. The salivary cortisol concentrations did not differ between the breakfasts at any time points measured (before and after WS2; Table 5).

\section{Discussion}

In the present study, we compared the effects of having either a breakfast drink containing protein from whey or carbohydrate from maltodextrin or having a control drink with a very low energy content on coping with mental workload. The breakfast drink with whey protein or carbohydrates significantly attenuated the increase in heart rate during the WS compared with the control drink. In addition, carbohydrates reduced subjective sleepiness during the WS, whereas whey protein increased the feeling of well-being when compared with the control drink.

The present study demonstrated that healthy subjects benefit from both whey protein and carbohydrates at breakfast time following a continuous and challenging work performance. We did not, however, find significant differences in cognitive performance (working memory, visuospatial working memory and divided attention) measured with dual and multitasks. Also, previous studies showed only modest 
Table 4. Heart rate (beats/min) recorded at rest before and after the Brain@Work (B@W) multitask test and during the B@W multitask test with the breakfast drinks of high protein, high carbohydrate and control in a $3 \times 3$ cross-over trial $(n 10)$

(Medians, interquartile ranges (IQR), minimum and maximum values)

\begin{tabular}{|c|c|c|c|c|}
\hline & High protein & High carbohydrate & Control & $P($ Friedman's ANOVA $)$ \\
\hline \multicolumn{5}{|l|}{ Before (at rest) } \\
\hline Median & $62 \cdot 6^{*}$ & $61 \cdot 5^{\star}$ & $60 \cdot 0$ & \multirow{3}{*}{0.03} \\
\hline IQR & $60 \cdot 9-68 \cdot 6$ & $59 \cdot 0-67 \cdot 8$ & $57 \cdot 7-62 \cdot 6$ & \\
\hline Minimum-maximum & $55 \cdot 2-75 \cdot 8$ & $58 \cdot 1-71 \cdot 2$ & $53 \cdot 5-67 \cdot 2$ & \\
\hline \multicolumn{5}{|l|}{ During B@W } \\
\hline Median & 68.5 & 66.5 & $64 \cdot 7$ & \multirow[t]{3}{*}{0.5} \\
\hline IQR & $63 \cdot 0-71 \cdot 8$ & $62 \cdot 5-70 \cdot 1$ & $63 \cdot 5-71 \cdot 7$ & \\
\hline Minimum-maximum & $54 \cdot 7-79 \cdot 3$ & $58 \cdot 2-83 \cdot 3$ & $62 \cdot 0-84 \cdot 7$ & \\
\hline \multicolumn{5}{|l|}{ After (at rest) } \\
\hline Median & 64.5 & $65 \cdot 1$ & $64 \cdot 1$ & \multirow[t]{3}{*}{0.9} \\
\hline IQR & $60 \cdot 5-70 \cdot 7$ & $61 \cdot 5-68 \cdot 3$ & $61 \cdot 7-68 \cdot 8$ & \\
\hline Minimum-maximum & $55 \cdot 6-73 \cdot 4$ & $56 \cdot 0-69.5$ & $59.5-73.9$ & \\
\hline \multicolumn{5}{|l|}{ Change (during-before) } \\
\hline Median & $2 \cdot 7^{\star}$ & $1.9^{*}$ & $7 \cdot 2$ & \multirow[t]{3}{*}{0.03} \\
\hline IQR & $0.8-7.7$ & $-1.5-5.3$ & $3.9-9.6$ & \\
\hline Minimum-maximum & $-7.5-10.4$ & $-4 \cdot 3-11 \cdot 0$ & $3.0-18.5$ & \\
\hline \multicolumn{5}{|l|}{ Change (after-during) } \\
\hline Median & $-2 \cdot 1$ & $-1 \cdot 8$ & $-1 \cdot 7$ & \multirow{3}{*}{0.5} \\
\hline IQR & $-4.6-1.0$ & -2.6 to -1.8 & $-4.7-0.6$ & \\
\hline Minimum-maximum & $-7.0-1.4$ & $-4 \cdot 4-4 \cdot 3$ & $-16 \cdot 8-4 \cdot 7$ & \\
\hline
\end{tabular}

* Median values were significantly different in pairwise comparison with the control breakfast $(P<0.05)$.

effects of a diet rich in Trp on cognition in healthy subjects. The clearest support is on memory functions, but there are no data on Trp effects on divided attention ${ }^{(9)}$. In addition, in healthy subjects, it is not clear whether a breakfast meal elicits beneficial effects on cognition when compared with a no-breakfast condition ${ }^{(6)}$. In the present study, differing the breakfast conditions affected coping with workload otherwise. During the WS, subjects were less fatigued after the breakfast high in carbohydrates when compared with the control breakfast. The finding is in agreement with an earlier study in which a glucose drink increased vigour compared with a non-energy drink before and during a short-term but severe stress

Table 5. Plasma ratio of tryptophan (Trp) to other large neutral amino acids (LNAA) before and $2 \mathrm{~h}$ after the high-protein $(\mathrm{HP})$, high-carbohydrate $(\mathrm{HC})$ and control breakfast drinks and salivary cortisol before and after work simulation 2 (WS2) in a $3 \times 3$ cross-over trial $(n 10)$

(Medians, interquartile ranges (IQR), minimum and maximum values)

\begin{tabular}{|c|c|c|c|c|}
\hline & $\mathrm{HP}$ & $\mathrm{HC}$ & Control & $P$ (Friedman's ANOVA $)$ \\
\hline \multicolumn{5}{|l|}{ Trp:LNAA $(\mu \mathrm{mol} / \mathrm{l}) /(\mu \mathrm{mol} / \mathrm{l})$} \\
\hline \multicolumn{5}{|l|}{ Before breakfast } \\
\hline Median & $0 \cdot 10$ & 0.10 & 0.11 & \multirow[t]{3}{*}{0.67} \\
\hline IQR & $0.09-0.12$ & $0.09-0.11$ & $0.09-0.12$ & \\
\hline Minimum-maximum & $0.09-0.14$ & $0.08-0.13$ & $0.09-0.14$ & \\
\hline \multicolumn{5}{|l|}{ After breakfast } \\
\hline Median & 0.13 & 0.13 & $0 \cdot 10$ & \multirow[t]{3}{*}{$<0.001$} \\
\hline IQR & $0.11-0.16$ & $0.10-0.15$ & $0.09-0.12$ & \\
\hline Minimum-maximum & $0 \cdot 10-0.17$ & $0.01-0.15$ & $0.08-0.12$ & \\
\hline \multicolumn{5}{|l|}{ Change } \\
\hline Median & 0.03 & 0.03 & 0.00 & \multirow[t]{3}{*}{$<0.001$} \\
\hline IQR & $0.02-0.05$ & $0.02-0.04$ & $-0.01-0.01$ & \\
\hline Minimum-maximum & $0.01-0.06$ & $0.01-0.06$ & $-0.02-0.02$ & \\
\hline \multicolumn{5}{|l|}{ Cortisol (nmol/l) } \\
\hline \multicolumn{5}{|l|}{ Before WS2 } \\
\hline Median & 3.0 & $2 \cdot 4$ & $4 \cdot 3$ & \multirow[t]{3}{*}{0.20} \\
\hline IQR & $2.08-4.75$ & $2 \cdot 15-5 \cdot 15$ & $2.98-8.00$ & \\
\hline Minimum-maximum & $1 \cdot 8-10 \cdot 1$ & $2 \cdot 0-6 \cdot 1$ & $2 \cdot 7-16 \cdot 4$ & \\
\hline \multicolumn{5}{|l|}{ After WS2 } \\
\hline Median & $2 \cdot 3$ & $2 \cdot 4$ & $3 \cdot 2$ & \multirow[t]{3}{*}{0.82} \\
\hline IQR & $1.7-4.7$ & $1.6-3.7$ & $1 \cdot 8-4.7$ & \\
\hline Minimum-maximum & $1.1-5.9$ & $1 \cdot 2-4.3$ & $1 \cdot 1-18 \cdot 1$ & \\
\hline \multicolumn{5}{|l|}{ Change } \\
\hline Median & -0.7 & -0.8 & $-1 \cdot 1$ & \multirow[t]{3}{*}{0.91} \\
\hline IQR & $-1.7-0.2$ & $-1.5-0.2$ & $-1.9-0.5$ & \\
\hline Minimum-maximum & $-4 \cdot 2-1 \cdot 8$ & $-3.5-2 \cdot 1$ & $-3 \cdot 7-5 \cdot 7$ & \\
\hline
\end{tabular}


exposure $^{(13)}$. It thus seems that the effects of carbohydrates are not exclusive for severe stress, but can improve mood before stress onset ${ }^{(13)}$ and during work-like tasks when compared with placebo.

In addition to decreased sleepiness, the nutritive breakfast seemed to promote a positive change in subjective wellbeing: the breakfast drink high in whey protein increased the feeling of well-being (pleased, relaxed, vigour and satisfied) when compared with the control drink. An earlier study by Boelsma et ll $^{(30)}$ also assessed postprandial wellness in healthy subjects and showed that in non-stress conditions, a meal high in whey protein increases subjective well-being 3$4 \mathrm{~h}$ after the meal when compared with a high-carbohydrate meal. The postprandial well-being effects of the whey protein meal were explained with higher cholekystokinin and lower ghrelin plasma concentrations ${ }^{(30)}$. The present study showed a positive influence of whey protein on postprandial wellbeing during the WS $1 \mathrm{~h}$ after the breakfast, and we can only speculate the role of metabolic responses of the breakfast drinks since gut hormones, for instance, were not studied. Studies on the effects of whey protein on stress have mostly shown results in the reduction of negative feelings in stressvulnerable subjects ${ }^{(14,16)}$ or with large changes in the Trp:LNAA ratio ${ }^{(38,39)}$

The mental workload index, NASA-TLX, is the most used and best known among questionnaires assessing subjectively experienced mental workload in several study fields ${ }^{(40)}$. However, as far as we know, there are no previous studies investigating the effect of the diet on mental workload using the NASA-TLX. In the present study, after the intake of the non-nutritive control drink, work-like dual and multitasks were considered the most loading. However, the difference compared with the HP and HC breakfasts was not significant, and there were also a large individual variation in NASA-TLX results, which is often seen with measures based on subjective questionnaire scales.

Heart rate and heart rate variability are widely used as indicators of physiological stress ${ }^{(35)}$. Following the control drink, the subjects' heart rates increased more in the WS with challenging work-like multitasks compared with the carbohydrate and protein drink conditions, suggesting that the HP and HC breakfasts may attenuate physiological stress occurring during a demanding work day. The results from the salivary cortisol analyses show that the WS did not cause serious stress to healthy subjects. Although salivary cortisol is a routinely used biomarker of psychological stress, there are several possible sources of variance that may affect this measure ${ }^{(41)}$, and the findings from studies investigating the associations between workplace stress and cortisol secretion are inconsistent ${ }^{(42)}$. In earlier studies showing a decrease in cortisol levels with diet manipulation, study designs were planned to cause serious stress and subjects were stress-vulnerable ${ }^{(16)}$.

In the present study, we measured mood and subjective workload alongside objective cognitive and physiological measures. There are some factors that may account for the results. First of all, the $30 \%$ increase in plasma Trp:LNAA observed after the carbohydrate and whey protein drinks when compared with the control drink may not have been sufficient for more pronounced study outcomes in subjects who were not necessarily stress-prone (this was not tested) and in design where the acute stress test was not used. In earlier interventions, which have shown improvement in stress performance or mood, whey protein-derived $\alpha$-lactalbumin has been compared with another milk protein, casein $^{(16,17,18)}$. Casein decreases the Trp:LNAA-ratio, which influences negatively stress performance when compared with $\alpha$-lactalbumin ${ }^{(16)}$. Some studies have shown that the Trp:LNAA ratio should increase $40-50 \%$ in order to induce a sufficient change in Trp availability and 5-HT synthesis. It has also been suggested that this is not enough, but even greater changes may be needed to detect any behavioural changes ${ }^{(42)}$. In the present study, $\alpha$-lactalbumin was in its natural proportion in whey protein. Consequently, it may be that the $\alpha$-lactalbumin content in the protein drink was not high enough to cause larger differences in the Trp:LNAA ratio.

Second, the breakfasts provided to subjects in the present study were in liquid form. It may be that a solid breakfast would have had stronger effects on work performance due to slower gastric emptying ${ }^{(6)}$. Also, the individual habits may affect the results. It has been proposed that the macronutrient composition closest to that habitually consumed would result in best cognitive performance ${ }^{(5)}$. Thus, future studies may have an advantage of adapting the experimental breakfast to individual habits and preferences, such as energy content and texture.

Different macronutrients may differ in their time of influence $^{(43)}$. We observed the performance in multitasks $180 \mathrm{~min}$ post-ingestion. In previous studies, the clearest cognitive effect of differing breakfast qualities has been shown in the late morning $(130 \text { and } 200 \mathrm{~min})^{(12)}$, whereas in breakfast $v$. no-breakfast conditions, the effect was found at $60 \mathrm{~min}$ after the consumption. Dye et $a l^{(6)}$ reviewed that the peak response to diet-induced mood change occurs $120 \mathrm{~min}$ after the consumption. We assessed the mood at about $60 \mathrm{~min}$ after the breakfast.

In addition to timing, the difficulty level of the cognitive tasks and the cognitive domains measured need to be considered. According to Hoyland et al. ${ }^{(5)}$, it is difficult to conclude which specific cognitive domains are the most sensitive to nutritional manipulations at breakfast time, although there is most abundant support for effects on memory. Moreover, the effects of macronutrient manipulations have been the most evident in demanding cognitive performance settings ${ }^{(12)}$. We used dual and multitasks (B@W and SS), which have been shown to improve sensitivity compared with a single-task paradigm since subjects are forced to divide their attention, thereby increasing task demand. The subtasks of these work-like tests have been used in previous nutrition interventions $^{(12)}$, and dual and multitasks (SS and B@W) have been shown to be sensitive tests in sleep deprivation studies $^{(31)}$, having the capacity to detect changes in working memory and attention.

Mental workload depends on the demands of the tasks, the individual functional state of the brain and the physiology of the autonomous nervous system, which are affected by, for example, stress and alertness ${ }^{(36)}$. In the present study, 
the demands of the cognitive tasks were set at an individual level for each subject and were kept constant throughout all the three study sessions. The analysis showed that there were significant differences in multitask performance between the three study sessions so that performance in work-like tasks improved in every study session. Thus, it seems that the individual task level was not set high enough for a cross-over design, which made the WS less challenging than it was intended to be.

Finally, we studied ten young healthy subjects which is a challenging population since they are less sensitive to nutritional variables than subclinical populations (e.g. recovered depressed, stress-vulnerable, highly anxious or impulsive subjects or subjects with sleep complaints) studied in previous studies $^{(16-18,42,44-46)}$. In these populations, brain 5-HT levels and function are declined, causing 5-HT sensitisation that makes the brain more vulnerable to dietary Trp:LNAA alterations ${ }^{(16)}$. Also, healthy subjects may become vulnerable to nutritional alterations in continuous and mentally challenging work performance, but probably not as much as the subclinical population. The majority of the subjects in the present study were women, which may affect the results. It has been shown previously that the cortisol response to stress in women and men may differ ${ }^{(47)}$. Men show a larger cortisol response than women, and particularly if a woman is taking contraceptives. In the present study, three of the seven women used oral contraceptives. We did not perform detailed analysis on sex differences as the sample size in the present study was relatively small. In addition to sex, although the subjects were apparently similar (according to the exclusion and inclusion criteria), the results show that there were rather large inter-individual differences in responses to the different breakfasts.

\section{Conclusions}

We conclude that in healthy subjects, a nutritive breakfast drink high in whey protein or carbohydrates may reduce sleepiness, improve subjective well-being and reduce the effects of workload on heart rate compared with a non-nutritive breakfast, and may thus aid in good task performance.

\section{Acknowledgements}

The present study was funded by the Salwe Research Program for Mind and Body (TEKES - the Finnish Funding Agency for Technology and Innovation). The authors thank the Brain and Technology Team and research nurses at the Finnish Institute of Occupational Health for their support in carrying out the study, and Dr Sari Mustonen from Valio Limited for helping with the study design and for providing the whey and maltodextrin powders. The authors also thank all the volunteers for their participation.

All the authors designed the research. N. S., A. Henelius and K. Pettersson conducted the research. N. S. and T. P. analysed the data. N. S. wrote the manuscript; K. Peuhkuri had primary responsibility for the final content. All the authors read and approved the manuscript. A. T. is employed by Valio Limited. None of the other authors has conflicts of interest.

\section{References}

1. Gopher D \& Donchin E (1986) Workload - an examination of the concept. In Handbook of Perception and Human Performance. Cognitive Processes and Performance, vol. II, pp. 41.41-41.49 [KR Boff, L Kaufman and JP Thomas, editors]. New York: A Wiley-Interscience Publication.

2. Yurko YY, Scerbo MW, Prabhu AS, et al. (2010) Higher mental workload is associated with poorer laparoscopic performance as measured by the NASA-TLX tool. Simul Healthc 5, 267-271.

3. Fischer K, Colombani PC, Langhans W, et al. (2002) Carbohydrate to protein ratio in food and cognitive performance in the morning. Physiol Behav 75, 411-423.

4. Kaplan RJ, Greenwood CE, Winocur G, et al. (2001) Dietary protein, carbohydrate, and fat enhance memory performance in the healthy elderly. Am J Clin Nutr 74, 687-693.

5. Hoyland A, Dye L \& Lawton CL (2009) A systematic review of the effect of breakfast on the cognitive performance of children and adolescents. Nutr Res Rev 22, 220-243.

6. Dye L, Lluch A \& Blundell JE (2000) Macronutrients and mental performance. Nutrition 16, 1021-1034.

7. Green MW, Elliman NA \& Rogers PJ (1995) Lack of effect of short-term fasting on cognitive function. J Psychiatr Res 29, $245-253$.

8. Markus CR (2008) Dietary amino acids and brain serotonin function; implications for stress-related affective changes. Neuromolecular Med 10, 247-258.

9. Silber BY \& Schmitt JAJ (2010) Effects of tryptophan loading on human cognition, mood, and sleep. Neurosci Biobehav Rev 34, 387-407.

10. Le Floc'h N, Otten W \& Merlot E (2011) Tryptophan metabolism, from nutrition to potential therapeutic applications. Amino Acids 41, 1195-1205.

11. Gilsenan MB, de Bruin EA \& Dye L (2009) The influence of carbohydrate on cognitive performance: a critical evaluation from the perspective of glycaemic load. Br J Nutr 101, 941-949.

12. Hoyland A, Lawton CL \& Dye L (2008) Acute effects of macronutrient manipulations on cognitive test performance in healthy young adults: a systematic research review. Neurosci Biobehav Rev 32, 72-85.

13. Markus CR (2007) Effects of carbohydrates on brain tryptophan availability and stress performance. Biol Psychol 76, 83-90.

14. Markus CR, Panhuysen G, Tuiten A, et al. (1998) Does carbohydrate-rich, protein-poor food prevent a deterioration of mood and cognitive performance of stress-prone subjects when subjected to a stressful task? Appetite 31, 49-65.

15. Markus R, Panhuysen G, Tuiten A, et al. (2000) Effects of food on cortisol and mood in vulnerable subjects under controllable and uncontrollable stress. Physiol Behav 70, 333-342.

16. Markus CR, Olivier B, Panhuysen GE, et al. (2000) The bovine protein alpha-lactalbumin increases the plasma ratio of tryptophan to the other large neutral amino acids, and in vulnerable subjects raises brain serotonin activity, reduces cortisol concentration, and improves mood under stress. Am J Clin Nutr 71, 1536-1544.

17. Markus CR, Olivier B \& de Haan EH (2002) Whey protein rich in alpha-lactalbumin increases the ratio of plasma tryptophan to the sum of the other large neutral amino acids and improves cognitive performance in stressvulnerable subjects. Am J Clin Nutr 75, 1051-1056.

18. Booij L, Merens W, Markus CR, et al. (2006) Diet rich in alpha-lactalbumin improves memory in unmedicated 
recovered depressed patients and matched controls. J Psychopharmacol 20, 526-535.

19. Lemmens SG, Born JM, Martens EA, et al. (2011) Influence of consumption of a high-protein $v s$. high-carbohydrate meal on the physiological cortisol and psychological mood response in men and women. PLoS One 6, e16826.

20. Lemmens SG, Martens EA, Born JM, et al. (2011) Lack of effect of high-protein $v s$. high-carbohydrate meal intake on stress-related mood and eating behavior. Nutr J 10, 136.

21. Meikle A, Riby LM \& Stollery B (2004) The impact of glucose ingestion and gluco-regulatory control on cognitive performance: a comparison of younger and middle aged adults. Hum Psychopharmacol 19, 523-535.

22. Ingwersen J, Defeyter MA, Kennedy DO, et al. (2007) A low glycaemic index breakfast cereal preferentially prevents children's cognitive performance from declining throughout the morning. Appetite 49, 240-244.

23. Hockey GR (1997) Compensatory control in the regulation of human performance under stress and high workload; a cognitive-energetical framework. Biol Psychol 45, 73-93.

24. Holm A, Lukander K, Korpela J, et al. (2009) Estimating brain load from the EEG. ScientificWorldJournal 9, 639-651.

25. Haavisto M-L, Porkka-Heiskanen T, Hublin C, et al. (2010) Sleep restriction for the duration of a work week impairs multitasking performance. J Sleep Res 19, 444-454.

26. Paturi M, Tapanainen H, Reinivuo H, et al. (2008) The National FINDIET 2007 Survey, pp. 29-31. Helsinki: National Public Health Institute. No. B23. http://www.julkari.fi/bitstream/ handle/10024/78088/2008b23.pdf?sequence=1 (accessed May 2010).

27. Hart SG \& Staveland LE (1988) Development of NASA-TLX (Task Load Index): results of empirical and theoretical research. In Human Mental Workload, pp. 239-250 [PA Hancock and N Meshkati, editors]. Amsterdam: North Holland Press.

28. Ảkerstedt T \& Gillberg M (1990) Subjective and objective sleepiness in the active individual. Int J Neurosci 52, 29-37.

29. Hänninen H (1989) Neurotoksisten haittojen seulonta, oirekyselyt ja psykologiset testit (The screening of neurotoxic effects, questionnaires and psychological tests). In Työterveyslaitoksen julkaisuja, vol. 16, pp. 36-37. Helsinki: Finnish Institute of Occupational Health.

30. Boelsma E, Brink EJ, Stafleu A, et al. (2010) Measures of postprandial wellness after single intake of two proteincarbohydrate meals. Appetite 54, 456-464.

31. Sallinen M, Holm J, Hirvonen K, et al. (2008) Recovery of cognitive performance from sleep debt: do a short rest pause and a single recovery night help? Chronobiol Int 25, 279-296.

32. Kane MJ, Hambrick DZ, Tuholski SW, et al. (2004) The generality of working memory capacity: a latent-variable approach to verbal and visuospatial memory span and reasoning. J Exp Psychol Gen 133, 189-217.

33. Elsmore TF (1994) SYNWORK1: a PC-based tool for assessment of performance in a simulated work environment. Behav Res Meth 26, 421-426.
34. Henelius A, Hirvonen K, Holm A, et al. (2009) Mental workload classification using heart rate metrics. Conf Proc IEEE Eng Med Biol Soc 2009, 1836-1839.

35. Stuiver A, de Waard D, Brookhuis KA, et al. (2012) Shortterm cardiovascular responses to changing task demands. Int J Psychophysiol 85, 153-160.

36. Niskanen J, Tarvainen M, Ranta-Aho O, et al. (2004) Software for advanced HRV analysis. Comput Methods Programs Biomed 76, 73-81.

37. Holm A (2010) Developing neurophysiological metrics for the assessment of mental workload and the functional state of the brain. PhD Thesis, Aalto University.

38. Markus CR, Firk C, Gerhardt C, et al. (2008) Effect of different tryptophan sources on amino acids availability to the brain and mood in healthy volunteers. Psychopharmacol 201, 107-114.

39. Markus CR, Verschoor E, Firk C, et al. (2010) Effect of tryptophan-rich egg protein hydrolysate on brain tryptophan availability, stress and performance. Clin Nutr 29, 610-616.

40. Noyes JM \& Bruneau DP (2007) A self-analysis of the NASA-TLX workload measure. Ergonomics 50, 514-519.

41. Hellhammer DH, Wüst S \& Kudielka BM (2009) Salivary cortisol as a biomarker in stress research. Psychoneuroendocrinol 34, 163-171.

42. Chandola T, Heraclides A \& Kumari M (2010) Psychophysiological biomarkers of workplace stressors. Neurosci Biobehav Rev 35, 51-57.

43. Firk C \& Markus CR (2009) Mood and cortisol responses following tryptophan-rich hydrolyzed protein and acute stress in healthy subjects with high and low cognitive reactivity to depression. Clin Nutr 28, 266-271.

44. Jones EK, Sünram-Lea SI \& Wesnes KA (2012) Acute ingestion of different macronutrients differentially enhances aspects of memory and attention in healthy young adults. Biol Psychol 89, 477-486.

45. Merens W, Booij L, Markus R, et al. (2005) The effects of a diet enriched with alpha-lactalbumin on mood and cortisol response in unmedicated recovered depressed subjects and controls. Br J Nutr 94, 415-422.

46. Markus CR, Jonkman LM, Lammers JH, et al. (2005) Evening intake of alpha-lactalbumin increases plasma tryptophan availability and improves morning alertness and brain measures of attention. Am J Clin Nutr 81, 1026-1033.

47. Verschoor E, Finlayson G, Blundell J, et al. (2010) Effects of an acute alpha-lactalbumin manipulation on mood and food hedonics in high- and low-trait anxiety individuals. Br J Nutr 104, 595-602.

48. Kirschbaum C, Kudielka BM, Gaab J, et al. (1999) Impact of gender, menstrual cycle phase, and oral contraceptives on the activity of the hypothalamus-pituitary-adrenal axis. Psychosom Med 61, 154-162. 